\title{
VEXAS: A Non - Systematic Literature Review
}

\author{
Hareesha Bharadwaj $^{1}$, Archisha Marya ${ }^{2}$, Janani Viswanathan $^{3}$, and Suraj Gulati ${ }^{4}$ \\ ${ }^{1}$ The University of Manchester Faculty of Biology Medicine and Health \\ ${ }^{2}$ University of Liverpool Faculty of Health and Life Sciences \\ ${ }^{3}$ Queen's University Belfast Faculty of Medicine Health and Life Sciences \\ ${ }^{4}$ University Hospitals Bristol and Weston NHS Foundation Trust
}

March 14, 2021

\begin{abstract}
Aim: Through this review, the authors intend to accumulate existing knowledge of VEXAS for referral, and to serve as an inspiration for further discovery, funding and research into the discipline. Methods: The non systematic literature review was conducted in January 2021, using Google Scholar and PubMed as the major extensive search engines. The keyword 'VEXAS' was used to narrow search results, and data was restricted to only those articles published in English. Results: The search, followed by the selection for relevancy led to a total of 5 pieces of literature being used for the purpose of this review; the lack of a huge number of results arises from the fact that the disease has been recently discovered. Discussion: Adult-onset inflammatory conditions are of increasing interest to medical professionals, and a number of patients with these conditions present with symptoms for which a concrete diagnosis is difficult to establish. In recent times, using an unconventional, yet remarkably effective genotype - based approach, researchers at the NIH have been able to discover a number of somatic mutations in UBA 1, which give rise to a unique disease. The disease, which has been named VEXAS (vacuoles, E1 enzyme, $\mathrm{X}$-linked, autoinflammatory and somatic syndrome) by the founders, arises from specific somatic mutations in the UBA 1 gene, and patients presenting with VEXAS have clinical manifestations - as sporadic fevers, chronic inflammation of the lungs and cartilage, and atypical vacuoles in myeloid cells, venous thromboembolism, ear and nose chondritis and macrocytic anaemia. VEXAS stems from accumulated somatic mutations in UBA 1, typically manifesting in three major variants, severely impairing the natural ubiquitylation process in cells, and shows no observable pattern of inheritance, according to the preliminary research conducted at NIH. Conclusion: Further study into VEXAS is needed for a better understanding of the syndrome.
\end{abstract}

Hareesha Rishab Bharadwaj (ORCID Number : 0000-0002-7979-2834)

Article Type : Non Systematic Review

Authors:

Hareesha Bharadwaj, Archisha Marya, Janani Viswanathan, Suraj Gulati

\section{VEXAS: A Non Systematic Literature Review}

\section{Authors}

\section{Hareesha Rishab Bharadwaj}

Student - First Year Medicine (MBChB)

Faculty of Biology, Medicine and Health

The University of Manchester

Oxford Road 
Manchester M13 9PL

ORCID Number : 0000-0002-7979-2834

\section{Archisha Marya}

Student - First Year Medicine (MBChB)

School of Medicine

Faculty of Health and Life Sciences

The University of Liverpool

Liverpool L69 3BX

ORCID Number : 0000-0002-2988-8045

\section{Janani Viswanathan}

Student - First Year Medicine (MBChB)

Faculty of Medicine, Health and Life Sciences

School of Medicine, Dentistry and Biomedical Sciences

Queen's University Belfast

Belfast BT9 7BL

\section{Suraj Gulati,}

Specialty Registrar - Trauma and Orthopaedics

Weston General Hospital,

First Floor Flat,

35 , St. John's Road

Clifton

Bristol BS8 2HD

Corresponding Author

\section{Hareesha Rishab Bharadwaj}

Student - First Year Medicine (MBChB)

Faculty of Biology, Medicine and Health

The University of Manchester

Oxford Road,

Manchester M13 9PL

Email : hareesha.bharadwaj@student.manchester.ac.uk

Telephone : +447827506465

Author Contributions

Hareesha Rishab Bharadwaj - Hareesha was involved in the majority of the data collection, analysis and data interpretation. He was involved in numerous hours of shortlisting articles relevant for this particular literature review, along with analysing them critically. 


\section{We would like to consider Hareesha as the first author.}

Archisha Marya - Archisha was involved in the drafting of the article, wording, and summarizing the vast amount of data that was collected by Hareesha. She was also primarily responsible for editing the article through the different rounds of inspection.

\section{We would like to consider Archisha as the first author as well.}

Janani Viswanathan - Janani was involved in critical revision of the paper. She was also involved in making edits, through the different rounds of inspection and rechecking.

We would like to consider Janani as the first author as well.

Suraj Gulati - As the most experienced of the team, and as a full-time specialty registrar in Trauma and Orthopaedics, Mr. Suraj was involved in critical appraisal of the piece. As the member of the team with the most extensive clinical knowledge, his role as the supervisor was invaluable in the drafting of the piece.

We would like to consider Suraj as the senior author, due to his primary role in supervision.

VEXAS : A Non Systematic Literature Review

Abstract

Aim : Through this review, the authors intend to accumulate existing knowledge of VEXAS for referral, and to serve as an inspiration for further discovery, funding and research into the discipline.

Methods : The non systematic literature review was conducted in January 2021, using Google Scholar and PubMed as the major extensive search engines. The keyword 'VEXAS' was used to narrow search results, and data was restricted to only those articles published in English.

Results : The search, followed by the selection for relevancy led to a total of 5 pieces of literature being used for the purpose of this review; the lack of a huge number of results arises from the fact that the disease has been recently discovered.

Discussion : Adult-onset inflammatory conditions are of increasing interest to medical professionals, and a number of patients with these conditions present with symptoms for which a concrete diagnosis is difficult to establish. In recent times, using an unconventional, yet remarkably effective genotype - based approach, researchers at the NIH have been able to discover a number of somatic mutations in UBA 1, which give rise to a unique disease. The disease, which has been named VEXAS (vacuoles, E1 enzyme, X-linked, autoinflammatory and somatic syndrome) by the founders, arises from specific somatic mutations in the UBA 1 gene, and patients presenting with VEXAS have clinical manifestations - as sporadic fevers, chronic inflammation of the lungs and cartilage, and atypical vacuoles in myeloid cells, venous thromboembolism, ear and nose chondritis and macrocytic anaemia. VEXAS stems from accumulated somatic mutations in UBA 1 , typically manifesting in three major variants, severely impairing the natural ubiquitylation process in cells, and shows no observable pattern of inheritance, according to the preliminary research conducted at NIH.

Conclusion : Further study into VEXAS is needed for a better understanding of the syndrome.

Review Criteria

As mentioned previously in the Results section of the abstract, the data utilized for this piece of literature was obtained through PUBMED and GOOGLE SCHOLAR, using keyword 'VEXAS'. The resulting searches were then scrutinized for relevance, and finally 5 publications were utilized. Out of these, 4 were taken directly from PUBMED, and 1 was taken from GOOGLE SCHOLAR.

The detailed process of selection has been included in the 'Methods' Section of the review.

Message for the Clinic 
Through this article, we as the authors intended to gather and accumulate the recent data and finding regarding the VEXAS disease upto the date of submission. VEXAS is a disorder that demands attention, and the current lack of knowledge/treatment makes the control of the disease impossible.

We hope our piece would be of benefit to other esteemed researchers and clinicians across the world, for utilization and reference as and when needed.

\section{Introduction}

During the latter half of 2020, researchers at the National Institutes of Health (NIH) reported a novel inflammatory disorder, aptly named as VEXAS (vacuoles, E1 enzyme, X-linked, autoinflammatory and somatic syndrome). The disease, which unites evidently unassociated inflammatory conditions, was discovered using an unorthodox genotype-based approach - it was recognized by clinical exome sequencing, with little attention to physical phenotypical manifestations or family history. (1)

Allergic and rheumatic diseases with genetic linkages have been traditionally identified using targeted sequencing approaches (2), and the discovery of VEXAS could potentially open up a new domain of research, encouraging genome sequencing to underpin the molecular basis of seemingly undiagnosable conditions, as was done in this case.

\section{Methods}

The pieces of literature used for study was obtained through a thorough search. The following databases were used for obtaining relevant articles:

\section{MEDLINE/PubMed}

\section{Google Scholar}

The Keywords used for the search on PubMed was 'VEXAS'. Owing to the very recent discovery of the disease, the search resulted in 5 results at the time of drafting the piece. 4 of these were selected for the degree of relevancy; these have been included in the citations section as (1), (3), (4), (5). Citation (2), was obtained from Google Scholar, with a search using the same keyword.

We acknowledge that the number of research articles available to us were limited owing to the very recent discovery of the disease.

Since the number of research articles available were limited, we did not choose to filter our search with MeSH terms.

The articles were chosen on the basis of specific factors -

- Degree of Relevance to the Disease

- Width and Breadth of information contained

- Clarity of Information contained

For the drafting of this literature review, we have utilized - 1 Original Study (Paper), 1 Editorial Piece, 1 Letter, 1 Non Systematic Literature Review, and 1 Case Report. This represents the breadth of articles selected to gather as much perspective as possible about the disease.

The articles were then scrutinized for relevance, initially by abstract, and then by thorough analysis and interpretation. We have limited our search to the articles published in English language only.

\section{The Original Study}

NIH's Undiagnosed Diseases Program was established in 2008, with the aim of investigating and providing a scientific rationale for diseases presented by patients for which a firm diagnosis was not possible to make. (3) Genetic data of 2560 individuals from this program were analysed, from which the first three male participants, identified as having missense mutations in codon 41 in UBA 1 were recognized. (4) 15 other men were also identified on the basis of correspondence in observational cohorts at the NIH Clinical Centre, and 
7 other men were also identified in similar study populations at the UCL and Leeds Teaching Hospital NHS Trusts in the UK. (1) Peripheral Blood Exome sequences in these 25 patients were isolated. These men were reported to have one of three somatic variants in methionine-41 (p.Met41Val, p.Met41Thr, p.Met41Leu) in UBA 1, which is a major E1 enzyme responsible for initiating the process of ubiquitylation. These mutations triggered a loss of the canonical cytoplasmic isoform of UBA 1 and caused mutant peripheral blood cells, which collectively activated self-attacking immune

pathways, reduced ubiquitylation, and promoted systemic inflammation. This learning was then used to unite clinical demonstrations, which have been discussed in the following sections. (1)

Characteristics of VEXAS

\section{Mutant Blood Cell Populations}

Patients with the UBA 1 mutations as discussed previously presented with an array of diversities within the blood cell populations. David D. Beck et al, who conducted the preliminary study, report the following observations in The New England Journal of Medicine, which have been summarized here. (1)

1. Lymphocytes ( $\mathrm{T}$ and $\mathrm{B}$ cells) were principally wildtype, but myeloid cells (neutrophils and monocytes) were mutant.

2. Mutant cells were obtained in early bone marrow progenitor cell populations, predominantly in MEPs (megakaryocytic erythroid progenitors), GMPs (granulocyte-monocyte progenitors), HPCs (hematopoietic progenitor cells) and multipotent progenitors.

3. Mutations were noticeably absent in mature lymphocytes.

4. Patients reported with decreased peripheral lymphocyte counts.

5. Mutations were absent in fibroblasts. (4)

\section{Effect on UBA 1 Function}

UBA 1 is the primary factor behind the production of ubiquitin-activating enzyme E1, which is essential for the activation of the ubiquitin-proteasome system. The gene encodes for a protein, which catalyses the first step in ubiquitination. This is a defence mechanism, marking unnecessary cellular proteins and stimulating their breakdown.

Usually, UBA has two notable isoforms -

UBA 1a - translation is initiated at p.Met1

UBA $1 b$ - translation is initiated at p.Met41

The mutations, causing the variants, described previously, lead to the formation of an unorthodox UBA 1c isoform, which arises from mutations at Met67.

UBA 1c isoform is hindered in its catalytic function.

The mutations resulting in the variants found in VEXAS favour the formation of the catalytically impaired UBA 1c isoform, over the catalytically proficient UBA $1 \mathrm{~b}$ isoform. (4)

\section{Physical Manifestations of UBA 1 Mutations and VEXAS}

Since the gene for UBA 1 is present on the X Chromosome, it is therefore justified that the frequency of VEXAS is substantially more in males as compared to females. Individuals who presented with these mutations displayed certain akin clinical features:

- Fever

- Ear and Nose Chondritis

- Pulmonary Infiltrate

- Skin Lesions

- Venous Thromboembolism 
- Abnormalities in Lung Function

- Chronic Inflammation of Lungs

- Inflammation of Cartilage

- Atypical Vacuoles in Myeloid Cells (4) (1) (5)

In addition to these, hematologic aberrations were also reported:

- Macrocytic Anaemia

- Thrombocytopenia

- Myeloid Dyspoiesis (1)

Please note that the primary source of the data for this section is David D. Beck et al's reporting in The New England Journal of Medicine.

\section{Immunological Manifestations of UBA 1 Mutations and VEXAS}

Specific shared gene expressions were noticed through an extensive study of peripheral blood samples obtained from these individuals. These gene expressions were responsible for the activation of a plethora of innate immune pathways, and was also responsible for the activation of inflammatory factors within these pathways. The findings from David D Beck et al's preliminary research have been summarised within this section for easy referral.

- Increased expression of Tumor Necrosis Factor

- Increased expression of Interleukin 6

- Increased expression of Interleukin 8

- Increased expression of Interferon Gamma

- Elevated levels of Serum Concentrations of C Reactive Protein

The observations from these patients (immunological and physical) met the diagnostic criteria for a variety conditions such as Myelodysplastic Syndrome, Giant Cell Arteritis, etc. (4) (1)

Pattern of Inheritance

The study conducted at NIH reported the following -

VEXAS does not result from numerical chromosomal abnormalities. This can be justified as the karyotype analysis of these patients did not reveal any chromosomal variations.

None of the affected men had affected relatives.

From these observations, researchers at NIH predict that VEXAS in not an inherited condition; the mutations causing the abnormalities should be somatic in nature.

The researchers also hypothesize that since these variants have a somatic origin, germline mutations affecting p.Met 41 should be lethal and non - compatible with life.

Since the gene for UBA 1 is present on the X chromosome, researchers also hypothesize that extra allele in women confers a protective function. At the time of writing, a case of VEXAS has not been found in women. (1)

\section{Treatment}

As of now, there have been no specific treatments for VEXAS. The study, conducted at NIH, involved administration of disease - modifying anti rheumatologic drugs, which unfortunately reported no favourable outcomes. The inflammatory symptoms in VEXAS, just as with other inflammatory conditions, could be dampened down with glucocorticoids. (1)

\section{Conclusion}

Through the means of this literature review, we as the authors aspire to provide an effective 
go - to article; a summary, of the clinical findings of VEXAS.

An increasing interest in adult-onset inflammatory conditions, in the recent years, have been noticed amongst rheumatologists. The innovative genotype first approach utilised in the discovery of VEXAS could be a motivation to incorporate similar study approaches to investigate other rheumatological conditions.

The authors encourage further funding, research and study into VEXAS. Further research into gene therapy is encouraged, which could potentially help to correct and adjust the somatic mutations described in this review article.

\section{Disclosure Statement}

The authors: Hareesha Rishab Bharadwaj, Archisha Marya, Janani Viswanathan and Suraj Gulati declare that no conflicting interests exist.

\section{Acknowledgements}

The authors would like to declare that no funding has been received for carrying out the study.

\section{Footnotes}

We would like to consider Hareesha Rishab Bharadwaj, Archisha Marya, and Janani Viswanathan as first authors, owing to their equal contributions in the drafting of the article. We would like to consider Suraj Gulati as the primary senior author, due to expertise provided and his role in supervision.

\section{Citations}

(1) -

Beck DB, Ferrada MA, Sikora KA, Ombrello AK, Collins JC, Pei W, Balanda N, Ross DL, Ospina Cardona D, Wu Z, Patel B, Manthiram K, Groarke EM, Gutierrez-Rodrigues F, Hoffmann P, Rosenzweig S, Nakabo S, Dillon LW, Hourigan CS, Tsai WL, Gupta S, Carmona-Rivera C, Asmar AJ, Xu L, Oda H, Goodspeed W, Barron KS, Nehrebecky M, Jones A, Laird RS, Deuitch N, Rowczenio D, Rominger E, Wells KV, Lee CR, Wang W, Trick M, Mullikin J, Wigerblad G, Brooks S, Dell'Orso S, Deng Z, Chae JJ, Dulau-Florea A, Malicdan MCV, Novacic D, Colbert RA, Kaplan MJ, Gadina M, Savic S, Lachmann HJ, Abu-Asab M, Solomon BD, Retterer K, Gahl WA, Burgess SM, Aksentijevich I, Young NS, Calvo KR, Werner A, Kastner DL, Grayson PC. Somatic Mutations in UBA1 and Severe Adult-Onset Autoinflammatory Disease. N Engl J Med. 2020 Dec 31;383(27):2628-2638. doi: 10.1056/NEJMoa2026834. Epub 2020 Oct 27. PMID: 33108101; PMCID: PMC7847551.

$(2)-$

Hoffman HM, Broderick L. Editorial: It Just Takes One: Somatic Mosaicism in Autoinflammatory Disease. Arthritis Rheumatol. 2017 Feb;69(2):253-256. doi: 10.1002/art.39961. PMID: 27748054; PMCID: PMC5538189.

$(3)-$

Gahl WA, Mulvihill JJ, Toro C, Markello TC, Wise AL, Ramoni RB, Adams DR, Tifft CJ; UDN. The NIH Undiagnosed Diseases Program and Network: Applications to modern medicine. Mol Genet Metab. 2016 Apr;117(4):393-400. doi: 10.1016/j.ymgme.2016.01.007. Epub 2016 Jan 22. PMID: 26846157; PMCID: PMC5560125.

(4) -

Onuora S. Somatic mutations cause VEXAS syndrome. Nat Rev Rheumatol. 2021 Jan;17(1):1. doi: 10.1038/s41584-020-00559-x. PMID: 33262468.

(5) - 
Fan BE, Cao L, Gallardo CA, Lee SMS, Koh LW, Goh LL, Ong KH, Kuperan P. Myeloid and lymphoid vacuolation in VEXAS syndrome. Am J Hematol. 2021 Jan 18. doi: 10.1002/ajh.26098. Epub ahead of print. PMID: 33460492. 